Volume 10, No.3, May - June 2021

International Journal of Advanced Trends in Computer Science and Engineering

Available Online at http://www.warse.org/IJATCSE/static/pdf/file/ijatcse701032021.pdf

https://doi.org/10.30534/ijatcse/2021/701032021

\title{
Key Benefits of Cloud-Based Internet of Vehicle (IoV)-Enabled Fleet Weight Management System
}

\author{
Muhammad Shakeel Faridi ${ }^{1}$, Dr. Saqib Ali ${ }^{2}$, Dr. Salman Afsar ${ }^{3}$, Zahid Javed ${ }^{4}$ \\ ${ }^{1,2,3,4}$ Department of Computer Science, University of Agriculture, Faisalabad, Pakistan \\ 12007ag51@uaf.edu.pk, ${ }^{2 *}$ saqib@uaf.edu.pk, ${ }^{3}$ ctdm@uaf.edu.pk, ${ }^{4}$ zahidjaved.uaf@gmail.com
}

\begin{abstract}
The fleet industry is flourishing at extreme levels with a wide acceptance of IoV-powered methods in various verticals. Fuel consumption, tire pressure readings, driver's driving habits, cargo monitoring, load measurement, and many other applications have now become possible in the fleet sector with the implementation of IoV and cloud based applications. Most of the industries heavily rely on transportation to carry loads and other different industrial products. The overall cost of vehicle maintenance involves a significant portion of total operating costs. Vehicle weight plays a major role in transporting goods from one place to the other. Many places have stringent rules regarding load transportation that only a certain amount of weight is allowed through a particular passage or road. This could result in heavy fines if all the regulations are not met and create unwanted expenses. It also creates unnecessary delays in product deliveries, which hampers the reputation of businesses. Hence, a smart solution can be integrated with the fleet sector to avoid extra costs during transit. In this paper, we will highlight the key benefits of IoV-based fleet weight management smart solution for fleet sector.
\end{abstract}

Key words : Cargo Monitoring, Cloud Applications, Fleet Industry, Internet of Vehicle (IoV),

\section{INTRODUCTION}

Internet of Things (IoT) / Internet of Vehicle (IoV) and Cloud Computing are booming. World is changing by the revolutionizing innovations of technology. Cloud Computing and $\mathrm{IoV}$ is an advanced technology that connects all the devices over the Internet. Collectively they form a secure analytical environment. It offers umpteen number of opportunities for industries to process smarter operations. While, on the other hand, cloud computing provides computing services like storage, database, software, networking and analytics over the Internet. The increasing trend of IoT technologies in industries such as automotive, manufacturing, healthcare and agriculture are actively driving the growth of this market. With the development of traditional manufacturing in the digital transformation, the cloud based IoT is driving the next industrial revolution of smart connectivity. The technology helps to improve the reduction in downtime and increase production efficiency.

The smart networks usually Includes a wide range of Internet of Things (IoT), including vehicles. In general, these interconnected vehicles form part of the Internet of Vehicles (IoV) network that interact with different IoT and IoV systems and fixed infrastructure to exchange information by using different protocol [1].

The Internet of Things is a centralized architecture in which all things/objects are connected via the Internet to interact with users and collect sensitive business information. Business systems can receive and analyze data to gain insights, and can manipulate the data through discrete decisions or automatic methods triggered by a set of specific conditions [2].

Cloud computing is a parallel and distributed system that includes networking groups of remote servers to allow centralized data storage, data processing tasks, and online access to IT services or resources [3],[4]. Using cloud computing, users can lease resources on monthly or annually basis instead of buying them. This way they can reduce the software cost [5], [6]. In addition, cloud computing provides on-demand services that customers can access from remote locations at any time. Unlike storing and retrieving data from 
a local computer, cloud computing allows the use of services from a remote computer [7], [8]. Customers use cloud services instead of working on their infrastructure, which means users don't need knowledge about network infrastructure.

A technical foundation of information can be developed by connectivity of hardware and software. To make the strong foundation of information, IoT are playing important role in every industry. IoT is a global physical interconnected network of multiple devices that relies on sensors, communication, networking, and information processing technology [9]. Smarter and smaller devices are being introduced in IoT domain like healthcare, supply chain, agriculture, logistics, transportation, automation, smart city, asset tracking, smart energy management, vehicle tracking etc [10]. [11] introduced a platform for control and monitoring of Wireless Sensor Network (WSN) in Textile Manufacturing Applications. This platform allowed users to connect any programming language's and new developed plug-ins. It also supported any different operating systems and designed GUI based interface that helped users to process live data from WSN.

IoT is a combination of different technologies Figure. 1 like wearable technology, industrial automation system, home appliances, smartphones, vehicles, and radio frequency identification (RFID) etc. By using these devices, we can communicate automatically with each other easily over the Internet due to unique identification of each device. These devices have capability to make decision without any assistance [12]. However, the data collected by IoT devices can contain both private and confidential information, and there are many security threats aimed at exploiting vulnerabilities in existing IoT infrastructure [13].

Different business application can be designed in IoT technology and it helps to make network smarter and more sophisticated. With the evolution and growing IoT business applications, a level of security threats can be increased which make the applications trustless for stakeholders [14]. To make it more reliable and trusted we need to design and implement a variety of security approaches to secure the IoT technology. The limitations of resources in IoT technology devices like memory, processing and energy limitations, an encryption algorithm implementation is a big challenge [15].

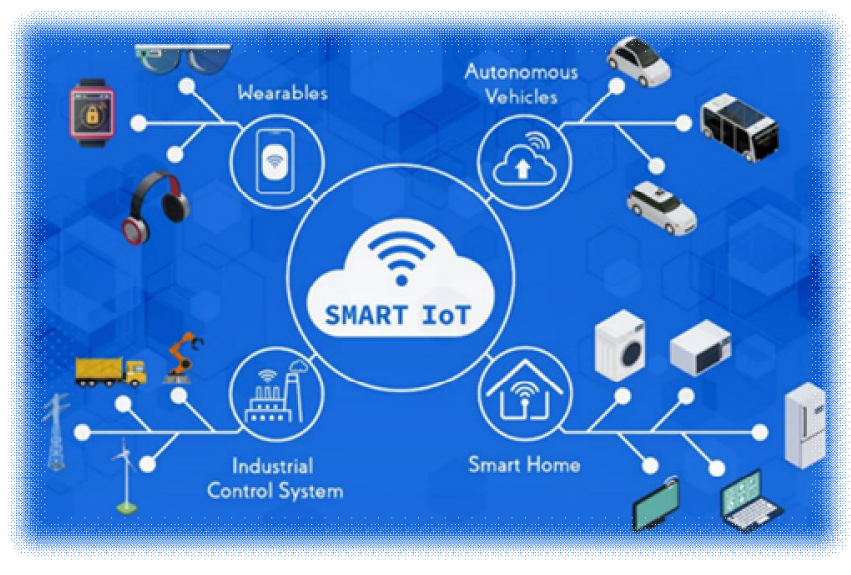

Figure 1: Smart IoT Architecture

Cloud based IoV-enabled applications and relevant technologies like global positioning system (GPS), radio frequency identification (RFID) can transform the fleet industry through digital information and real-time inspection and control. All these technologies have brought huge opportunities in the fleet industry, reshaping business activities and their real-time operating procedures by conducting real-time business analysis models Figure 2.

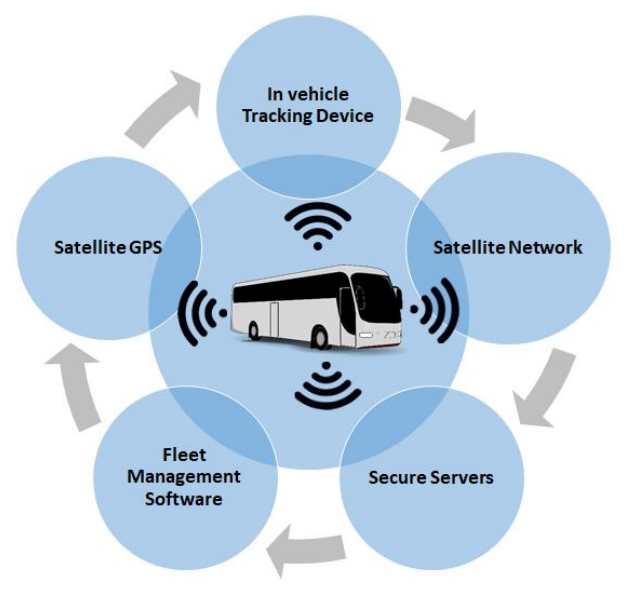

Figure 2: IoT-Enabled Vehicle

The cloud based IoV-enabled fleet weight management system offer many benefits to transporters like fuel management, vehicle tracking, route specification, driver safety, sensor based information, reduced accidents etc. it also provides facility to manage transport business under one umbrella by using gadgets. Our objective in this paper is to highlight the key benefits that fleet industry can get by implementing cloud based IoV-enabled smart fleet weight management system. This will help the transporter to boost their business by using this reliable and traceable system. It will also help to flourish the fleet industry. The paper is organized as follows. Section 2 is cloud based IoT integration. We discussed cloud based IoV-enabled fleet management 
system in Section 3. Section 4 highlights the key benefits and Section 5 concludes the paper.

\section{CLOUD BASED IOT INTEGRATION}

Cloud Computing services are implemented in many areas relevant to the IoT, including Genomics Data Processing, Vehicle Tracking System, Services for Small and Medium Businesses, E-Learning, Manufacturing, Emergency Recovery, Smart Cities and others, Hospitality Business, E-Government and Human Resource Administration [16]. Benefits in cloud computing and the IoT based application environments are unique [17]. Cloud based fleet IoV showing in Figure. 3 that the application communication for end user.

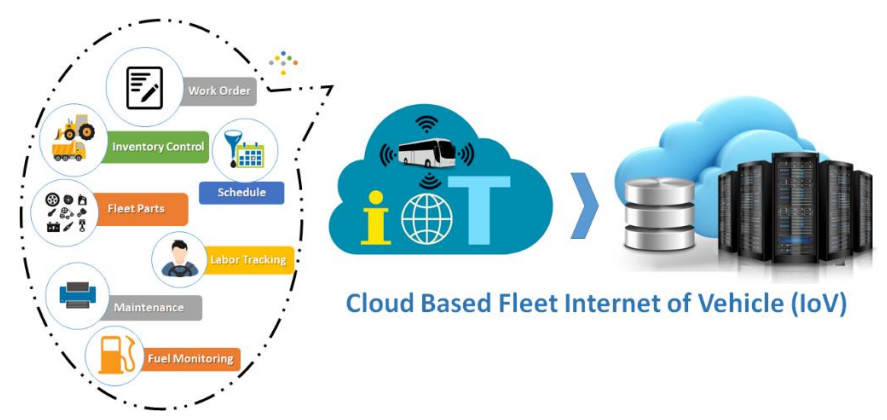

Figure 3: Cloud Based Fleet Internet of Vehicle (IoV)

Cloud computing and IoT have spread globally and expanded quickly in recent years. The characteristics they show can be outstanding when combined. They are special and important for each other [18]. A series of applications related to cloud and IoT architecture Figure. 4 are developed and accumulate data as it gets help from cloud storage and computing functions.

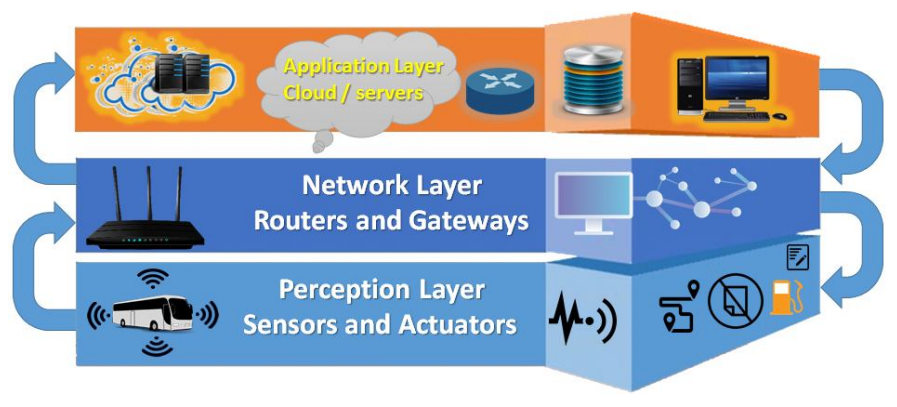

Figure 4: IoT Based Cloud Architecture

\section{CLOUD BASED IOV-ENABLED FLEET MANAGEMENT SYSTEM}

Fleet management benefits from the growth of cloud based IoV devices. IoV-based solutions provide opportunities to connect fleets that allows fleet managers to remotely understand their operations in detail to formulate better business strategies. The IoT and IoV brings multiple benefits and improvements in all areas of the economy. Today, most companies have turned to the cloud based IoT and IoV and are willing to invest in it. According to professionals, the Industrial IoT is in a leading position in terms of growth and development. The transportation industry is one of the industry for investing in the IoV. An effective transportation system can not only increase the productivity of an enterprise, but also increase its profitability. IoV applications can be used for personal and commercial vehicles, with functions ranging from traffic control to parking sensors, fault detectors, fuel consumption, tire condition, weight, and more. The key benefits as shown in Figure. 5 are important to understand the usage of IoV.

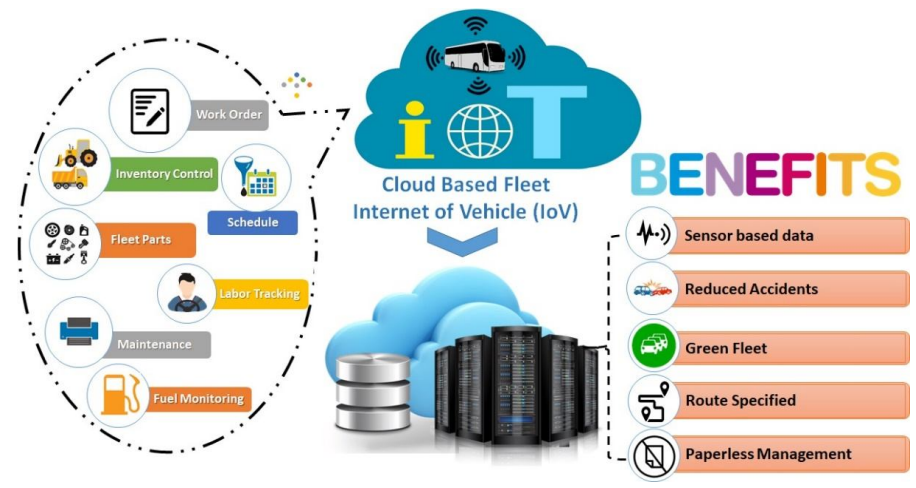

Figure 5: CLOUd BASED IOT-ENABLED KEY BENEFITS

\section{KEY BENEFITS}

In a fleet industry, weight management is one of the essentialities required before commencing with the transit process. If the cargo weight exceeds a certain limit, it can cause fines and also temporarily keep the driver's licenses at stake. This could result in heavy fines if all the regulations are not met and create unwanted expenses. It also creates unnecessary delays in product deliveries, which hampers the reputation of businesses. Most of the industries heavily rely on transportation to carry loads and other different products. The overall cost of vehicle maintenance involves a significant portion of total operating costs. Also, vehicle weight plays a major role in transporting goods from one place to the other. Many places have stringent rules regarding load transportation that only a certain amount of weight is allowed through a particular passage or road. Therefore, integrating a well-structured weight management system can be an intelligent decision to avoid such issues. The IoV-powered system is equipped with the latest load sensor devices and 
gateways, offering accuracy in weight readings and allowing you to meet the weight criteria throughout the journey. This encourages better business growth and avoids extra expenses.

\subsection{Sensor based data}

IoV technology is all about capturing data through sensors, and this has been a greater advantage for the fleet industry to upgrade itself in the most unique ways possible. Especially talking about the weights that the large fleeting trucks carry; it is an essential part of the transportation business. But what's more important is to comply with the regional guidelines. Therefore, load sensors play an important role in integrating IoT-based weight management with the transportation business. It is specially designed to provide control over fleet loads and cargo weights during transportation. The weight of the cargo is then saved as useful information on a cloud platform, which can be accessed remotely for analysis.

\subsection{Reduced Accidents}

Vehicles are bound to experience severe accidents due to over speeding, turn-by-turn directions, and product overload during the transit. With the help of an IoV-powered weight monitoring system, we can easily meet the load conditions required in a particular region. Also, it helps in raising the levels of industrial productivity by providing accurate product weight measurements. Smart weight measurement leads to the exact number of products being loaded on the fleet as per the guidelines. It thus results in enhanced safety of the vehicle as well as the driver. We can keep a genuine record of the load being added to the vehicle and get alerts in case of any disruption in the product order.

\subsection{Green Fleet}

There lies a direct correlation between greenhouse gas emissions and vehicle weight. Every extra amount added to the product in-transit requires more hard work from the engine and increases the amount of fuel consumed during the whole journey. It directly impacts the environment and increases the carbon footprint at greater costs. This calls for a smart system that enables the exact weighted measurement of the products and ensures safety along with complying with the strict regulations. The IoV-powered solution is the best way to uplift the industry due to its sensor capabilities and power-boosting functionality.

\subsection{Route Specification}

GPS based technology keeps the drivers on track during their journeys by specifying routes towards the destination through smart systems. IoV-based solutions are well-equipped with advanced analytics and algorithms to provide the drivers with forecasted route information and weather-related data to avoid delays. It helps them in reaching their destination on time and contributes very well to timely deliveries. Moreover, route optimization helps the fleet drivers and authorities to communicate well through a single platform, which makes it easier to optimize the schedules and products delivery whenever and as needed. It thus helps in keeping up with the stocks and allows you to replace the products on short notices, without hampering the delivery time.

\subsection{Paperless Management}

The IoV-based system works through smart devices, where the authorities browse all the options via a single platform. It involves no paperwork and encourages paperless management of fleet operations with the help of smart gadgets like mobiles, tablets, and desktops. The acceptance of the IoV technology is an incredible step taken towards a better and sustainable environment. It works on the internet which enables fleet functioning and cargo weight management super easy. It also allows remote management, where you can operate the entire functioning of the loading-unloading processes remotely and get every detail on the interconnected gadget.

\section{CONCLUSION}

Fleet management provides benefits to multiple industries. Typically, couriers, trade workers, repair and service businesses and oil and gas delivery will rely on fleet management. These industries depend on their work vehicles to provide a service. Fleet management ensures a smoother operation and efficient real-time customer service through database software, telematics and GPS tracking. The healthcare sector, such as pharmaceutical services, home health care, assisted living and elderly/disabled transport services also benefit from fleet management. Businesses in manufacturing, insurance, utilities and other corporate or sales services can formulate consistency and company growth through management policies as well. All these industries heavily rely on transportation to carry goods and other different products. The total cost of vehicle maintenance accounts for a large part of the total operating cost. Moreover, the weight of the vehicle plays an important role in the process of transporting goods from one place to another. 
Many places have strict regulations on the transportation of goods, that is, only a certain amount of weight is allowed through a specific passage or road. If you do not comply with all regulations, you may be fined and incurred unnecessary expenses. It will also cause unnecessary delays in the product delivery process, thereby affecting corporate reputation. In this paper, we highlight the key benefits that transporters can get after implementation of IoV Fleet weight management system to run their business smoothly. This work may be expanded in the future and we will propose to transfer Cloud-based Internet of Vehicle (IoV)-Enabled Fleet Weight Management System to blockchain environment

\section{REFERENCES}

[1] P. Bagga, A. K. Das, M. Wazid, J. J. P. C. Rodrigues, K.-K. R. Choo, and Y. Park, "On the Design of Mutual Authentication and Key Agreement Protocol in Internet of Vehicles-Enabled Intelligent Transportation System," IEEE Trans. Veh. Technol., vol. 70, no. 2, pp. 1736-1751, 2021.

[2] M. S. Faridi, S. Ali, G. Duan, and G. Wang, "Blockchain and IoT Based Textile Manufacturing Traceability System in Industry 4.0," in International Conference on Security, Privacy and Anonymity in Computation, Communication and Storage, 2020, pp. 331-344.

[3] L. M. Haji, S. R. Zeebaree, O. M. Ahmed, A. B. Sallow, K. Jacksi, and R. R. Zeabri, "Dynamic resource allocation for distributed systems and cloud computing," TEST Eng. Manag., vol. 83, pp. 22417-22426, 2020.

[4] P. Priyadarshinee, R. D. Raut, M. K. Jha, and B. B. Gardas, "Understanding and predicting the determinants of cloud computing adoption: A two staged hybrid SEM-Neural networks approach," Comput. Human Behav., vol. 76, pp. 341-362, 2017.

[5] S. Vakilinia, M. M. Ali, and D. Qiu, "Modeling of the resource allocation in cloud computing centers," Comput. Networks, vol. 91, pp. 453-470, 2015.

[6] O. Alzakholi, H. Shukur, R. Zebari, S. Abas, and M. Sadeeq, "Comparison among cloud technologies and cloud performance," J. Appl. Sci. Technol. Trends, vol. 1, no. 2, pp. 40-47, 2020.

[7] D. Kesavaraja and A. Shenbagavalli, "QoE enhancement in cloud virtual machine allocation using Eagle strategy of hybrid krill herd optimization," J. Parallel Distrib. Comput., vol. 118, pp. 267-279, 2018.

[8] S. R. Zeebaree, R. R. Zebari, and K. Jacksi, "Performance analysis of IIS10. 0 and Apache2 Cluster-based Web Servers under SYN DDoS Attack," TEST Eng. Manag., vol. 83, pp. 5854-5863, 2020.
[9] A. Mijuskovic, A. Chiumento, R. Bemthuis, A. Aldea, and P. Havinga, "Resource Management Techniques for Cloud/Fog and Edge Computing: An Evaluation Framework and Classification," Sensors, vol. 21, no. 5, p. 1832, 2021.

[10] Z. Bi et al., "Real-time force monitoring of smart grippers for Internet of Things (IoT) applications," $J$. Ind. Inf. Integr., vol. 11, pp. 19-28, 2018.

[11] P. Wang, S. Chaudhry, L. Li, Z. Bi, G. Wang, and L. $\mathrm{Da} \mathrm{Xu}$, "A visualization platform for internet of things in manufacturing applications," Internet Res., 2016.

[12] S. Ali, G. Wang, B. White, and K. Fatima, "Libra critique towards global decentralized financial system," in International Conference on Smart City and Informatization, 2019, pp. 661-672.

[13] F. A. Alaba, M. Othman, I. A. T. Hashem, and F. Alotaibi, "Internet of Things security: A survey," $J$. Netw. Comput. Appl., vol. 88, pp. 10-28, 2017.

[14] S. E. Tsigie and G. A. Dagnaw, "The Role of Robotics Technology and Internet of Things for Industry 4.0 Realization," Int. J., vol. 10, no. 2, 2021.

[15] R. Xu, C. Cheng, Y. Qin, and T. Jiang, "Lighting the way to a smart world: lattice-based cryptography for internet of things," arXiv Prepr. arXiv1805.04880, 2018.

[16] M. A. Sadeeq, A. I. Abdulla, A. S. Abdulraheem, and Z. S. Ageed, "Impact of Electronic Commerce on Enterprise Business," Technol. Rep. Kansai Univ, vol. 62, no. 5, pp. 2365-2378, 2020.

[17] H. Shukur, S. Zeebaree, R. Zebari, D. Zeebaree, O. Ahmed, and A. Salih, "Cloud computing virtualization of resources allocation for distributed systems," J. Appl. Sci. Technol. Trends, vol. 1, no. 3, pp. 98-105, 2020.

[18] A. Hosseinian-Far, M. Ramachandran, and C. L. Slack, "Emerging trends in cloud computing, big data, fog computing, IoT and smart living," in Technology for smart futures, Springer, 2018, pp. 29-40. 